\title{
Dados biométricos de frutos e sementes de Hymenaea courbaril var. stilbocarpa (Hayne) Y. T. Lee \& Langenh e H. martiana Hayne
}

\author{
Gabrielle Rodrigues Cunha-Silva ${ }^{1}$ \\ Christiane Moreira Rodrigues ${ }^{1}$ \\ Sabrina do Couto de Miranda ${ }^{2 *}$ \\ ${ }^{1}$ Curso de Ciências Biológicas, Universidade Estadual de Goiás \\ ${ }^{2}$ Departamento de Botânica, Universidade Estadual de Goiás \\ * Autor para correspondência \\ SQN 405, bl. G, ap. 107, CEP 70.846-070, Brasília - DF, Brasil \\ sabrina_miranda@yahoo.com.br
}

Submetido em 28/11/2011

Aceito para publicações em 10/05/2012

\section{Resumo}

O Cerrado apresenta alta biodiversidade de espécies, porém estimativas indicam que 48\% de sua vegetação já foram desmatados ou alterados. Diante deste cenário, há iminente demanda para recuperação de áreas degradadas, e estudos que visem conhecer a biometria das espécies nativas são relevantes para este propósito. Este trabalho objetivou caracterizar a biometria de frutos e sementes de Hymenaea courbaril var. stilbocarpa e H. martiana, e comparar as características biométricas de diferentes espécies do mesmo gênero para auxiliar na identificação taxonômica destas espécies. No entorno do município de Itapuranga - GO foram amostradas cinco árvores matrizes de $H$. courbaril var. stilbocarpa e três de $H$. martiana, destas foram coletados frutos, os quais foram medidos e pesados. As espécies em estudo se diferenciaram significativamente em relação ao comprimento dos frutos. Com relação às sementes, $H$. martiana apresentou parâmetros biométricos com os maiores valores. Além disso, frutos e sementes das duas espécies apresentaram pesos significativamente diferentes. A análise comparativa entre as espécies do gênero mostrou que $H$. intermedia pode ser facilmente diferenciada das demais por possuir frutos grandes, já H. martiana apresentou sementes grandes e robustas. As informações apresentadas são relevantes e ratificam a importância da biometria de frutos e sementes para diferenciação de espécies.

Palavras-chave: Biometria; Cerrado; Fabaceae; Jatobá

\section{Abstract}

Biometric data of fruits and seeds of Hymenaea courbaril var. stilbocarpa (Hayne) Y. T. Lee \& Langenh and $\boldsymbol{H}$. martiana Hayne. The Cerrado presents high species diversity, but estimates indicate that $48 \%$ of its vegetation has been cleared or changed. Based on this scenario there is imminent demand for the recovery of degraded areas, and studies that aim to understand the biometrics of native species are relevant for this purpose. The goal of this study was to characterize the biometry of fruits and seeds of Hymenaea courbaril var. stilbocarpa and $H$. martiana, and to compare the biometric characteristics of different species in this genus to help with their identification. Near the city of Itapuranga, in the state of Goiás, five tree matrixes of $H$. courbaril var. stilbocarpa and three of $H$. martiana were sampled. From each species, fruits were collected, measured and weighed. The species differed significantly in relation to fruit length. Regarding the seeds, $H$. 
martiana presented biometric parameters with the highest values. In addition, fruits and seeds of both species had significantly different weights. The comparative analysis of the genus showed that $H$. intermedia can be easily distinguished from other species because of its large fruits, and that $H$. martiana had the largest and most robust seeds. The information presented in this study is relevant and shows how the biometry of fruits and seeds can be used to differentiate species.

Key words: Biometrics; Cerrado; Fabaceae; Jatobá

\section{Introdução}

O Cerrado é o segundo maior bioma brasileiro em extensão geográfica (KLINK; MACHADO, 2005) e faz contato com a Caatinga, Floresta Atlântica, Floresta Amazônica e Pantanal (FELFILI; SILVA JÚNIOR, 2005). Originalmente, este ecossistema ocupava cerca de $20 \%$ do território nacional e sua distribuição é altamente coincidente com a região do Planalto Central brasileiro (OLIVEIRA-FILHO; RATTER, 2002; FELFILI; SILVA JÚNIOR, 2005). Abrangendo os estados de Goiás, Tocantins, além do Distrito Federal, e parte da Bahia, Maranhão, Mato Grosso, Mato Grosso do Sul, Minas Gerais, Paraná, Piauí e São Paulo (RIBEIRO; WALTER, 2008; SANO et al., 2008).

O Cerrado é um complexo vegetacional que compreende formações campestres, savânicas e florestais (FURLEY; RATTER, 1988; RIBEIRO; WALTER, 2008). Com base na variação na densidade dos estratos arbóreos e herbáceos, tipo de substrato e associação com os cursos d'água, Ribeiro e Walter (2008) descreveram para o Cerrado 11 tipos fitofisionômicos, a saber: campo limpo, campo sujo e campo rupestre; cerrado sentido restrito, palmeiral, vereda e campo de murundus; mata de galeria, mata ciliar, mata seca e cerradão. Esta alta heterogeneidade se reflete em grande diversidade, só para a flora já foram catalogadas mais de 11.000 espécies (MENDONÇA et al., 2008). Além disso, o Cerrado abriga 35,9\% da riqueza de plantas vasculares do Brasil (FORZZA et al., 2010), ratificando que sua flora é uma das mais ricas e diversas dentre as savanas mundiais (LEWINSOHN; PRADO, 2002; FELFILI et al., 2004).

Diante da alta heterogeneidade ambiental, o Cerrado, da mesma forma que a Floresta Amazônica, fornece importantes serviços ambientais ao Brasil e ao mundo, como a manutenção de biodiversidade, o ciclo hidrológico e o armazenamento de carbono (FEARNSIDE, 2008).

Apesar de sua expressiva importância, estimativas atuais indicam que $48 \%$ do Cerrado já foram desmatados ou alterados por usos antrópicos diversos (MMA/ IBAMA/PNUD, 2009). As principais formas de uso da terra são para as pastagens encontradas principalmente nos estados de Goiás, Minas Gerais e Mato Grosso do Sul, e para as culturas comerciais que ocupam significativas áreas de Goiás, Mato Grosso e São Paulo (SANO et al., 2010). Devido à pressão antrópica e mudança de uso da terra, o bioma é hoje considerado um dos 34 hotspots mundiais de biodiversidade (MITTERMEIER et al., 2005; RIBEIRO et al., 2005).

Diante deste cenário, no Cerrado há iminente demanda para recuperação de áreas degradadas, principalmente áreas de preservação permanentes ao longo dos cursos d'água (FELFILI et al., 2008) e de reservas legais (AQUINO et al., 2007), obedecendo ao Código Florestal vigente (Lei Federal $n^{\circ} 4.771,1965$ ). Assim, estudos que visem conhecer a biometria das espécies nativas são extremamente relevantes para a diferenciação das espécies em campo, tendo uma aplicação prática em trabalhos de coleta de diásporos para produção de mudas com o intuito de recuperação de áreas degradadas e arborização urbana.

Entre as espécies nativas podemos destacar o gênero Hymenaea L., pertencente à família Fabaceae, uma das mais representativas do Cerrado (MENDONÇA et al., 2008). As espécies do gênero Hymenaea ocorrem na Floresta Amazônica, Caatinga, Cerrado, Floresta Atlântica e Pantanal (RIBEIRO, 2010). O gênero engloba 14 espécies (W3 Tropicos), destas 12 ocorrem no Brasil, Hymenaea aurea Y.T. Lee \& Langenh., $H$. courbaril L., H. eriogyne Benth., H. intermedia Ducke, $H$. maranhensis Y.T.Lee \& Langenh., H. martiana Hayne, H. oblongifolia Huber, H. parvifolia Huber, H. 
reticulata Ducke, $H$. rubliflora Ducke, $H$. stigonocarpa Mart. ex Hayne e H. velutina Ducke (RIBEIRO, 2010).

Dentre as espécies mencionadas acima, $H$. courbaril var. stilbocarpa (Hayne) Y. T. Lee \& Langenh e H. martiana Hayne foram selecionadas para este estudo. Hymenaea courbaril var. stilbocarpa, conhecida como Jatobá-da-mata, é uma árvore com altura entre 15 e 20m (LORENZI, 2008) e H. martiana, conhecida como Jatobá-da-casca-fina, apresenta altura entre 8 e $16 \mathrm{~m}$ (observação pessoal). Hymenaea martiana apresenta folíolos menores e densamente pilosos na face abaxial (observação pessoal), já $H$. courbaril var. stilbocarpa apresenta folíolos maiores, glabros e lustrosos (SILVA JÚNIOR; PEREIRA, 2009). Estas espécies podem ser encontradas nas florestas estacionais no Cerrado (MATOS; QUEIROZ, 2009), quando estão isoladas são facilmente diferenciadas. Porém, em florestas nativas com dossel fechado, há grande dificuldade para visualização das copas, e consequentemente para coleta de material botânico e diferenciação destas espécies, sendo, na maioria das vezes, identificadas até gênero, tomando por base a presença de frutos que perduram no solo. Assim, este estudo teve por objetivos caracterizar a biometria de frutos e sementes e comparar tais características entre espécies do mesmo gênero.

\section{Material e Métodos}

As espécies estudadas foram coletadas no entorno do município de Itapuranga (15³3'40”S e 4956’33”W) que se localiza na região noroeste do estado de Goiás. O município apresenta área de $1.277,15 \mathrm{~km}^{2}$, altitude média de $600 \mathrm{~m}$, temperatura média anual $25^{\circ} \mathrm{C}$ e precipitação média de $1.600 \mathrm{~mm}$ anuais (COSTAJUNIOR; FIGUEIREDO, 2003).

Foram amostradas cinco árvores matrizes de $H$. courbaril var. stilbocarpa e três matrizes de H. martiana, conhecidas popularmente como Jatobá da Mata. Não foi possível amostrar número igual de matrizes, pois H. martiana apresenta baixa densidade na região (observação pessoal). Em cada matriz foram coletados em torno de 20 frutos maduros na época seca, no mês de junho de 2011. As coletas não foram realizadas imediatamente, portanto os frutos foram armazenados em local arejado à temperatura ambiente até o início das análises.

Dados biométricos (comprimento, largura e espessura) de 100 frutos de H. courbaril var. stilbocarpa e 45 frutos de $H$. martiana foram medidos com paquímetro. Para pesagem foram amostrados aleatoriamente 20 frutos de $H$. courbaril var. stilbocarpa e 15 frutos de $H$. martiana. Após esta etapa, os frutos foram quebrados com martelo e para separar a polpa das sementes estas permaneceram submersas em água por 24 horas.

Dentre as sementes com aspecto morfológico viável, foram escolhidas, aleatoriamente, 100 sementes de $H$. courbaril var. stilbocarpa e 30 sementes de $H$. martiana. Para estas sementes foram realizadas medições de comprimento, largura e espessura. Considerou-se comprimento a medida do ápice à base da estrutura em estudo, largura e espessura foram tomadas na região mediana da semente. Para a pesagem foram amostradas aleatoriamente 20 sementes de ambas as espécies em estudo. Todas as medidas biométricas foram realizadas utilizando-se paquímetro com precisão de milímetros e os pesos obtidos em balança de precisão.

Para cada uma das variáveis calculou-se a média aritmética e o desvio-padrão. Os parâmetros biométricos foram comparados por meio de teste $t$ de Student ( $p \leq$ $0,05)$ utilizando o programa JMP 9.0 (SAS Institute, Cary, North Carolina, USA).

\section{Resultados}

Os frutos das duas espécies de Hymenaea apresentaram coloração marrom escuro quando maduros (Figura 1A-B). As sementes de $H$. courbaril var. stilbocarpa possuem coloração predominante castanho claro a castanho escuro, porém também foram encontradas sementes alaranjadas (Figura 1C). Hymenaea martiana tem sementes castanho avermelhadas com porções mais claras (Figura 1D).

Quanto à forma, as espécies estudadas apresentaram sementes com forma predominantemente achatadas. Em H. courbaril var. stilbocarpa também foram encontradas sementes elipsoides, oblongas e arredondadas. Já em H. martiana as sementes também apresentaram 
FIGURA 1: Variação morfológica dos frutos (A) e sementes (C) de H. courbaril var. stilbocarpa e dos frutos (B) e sementes (D) de $H$. martiana.
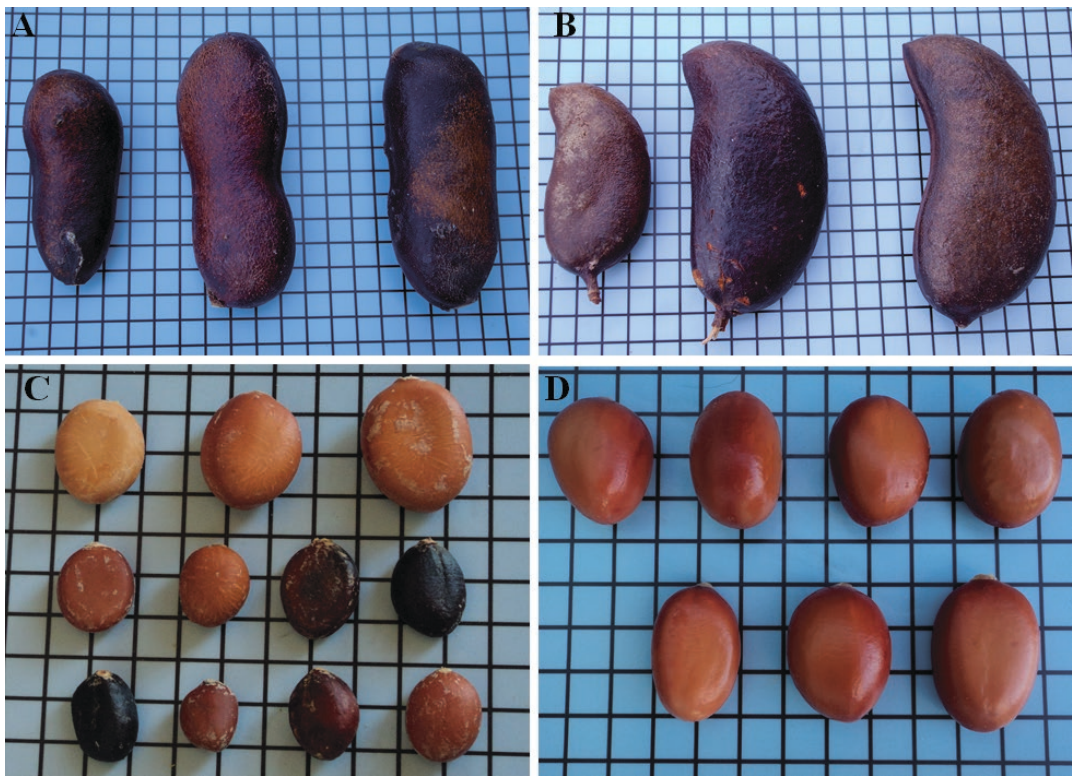

o formato arredondado e elíptico (Figura 1C e 1D, sementes por fruto, $48,9 \%$ dos frutos apresentaram respectivamente).

Em média, os frutos de H. courbaril var. stilbocarpa apresentaram $11,6 \mathrm{~cm}$ de comprimento, $4,9 \mathrm{~cm}$ de largura e $3,9 \mathrm{~cm}$ de espessura (Tabela 1 ). $\mathrm{O}$ número de sementes por fruto variou de uma a onze, $18 \%$ do total de frutos analisados apresentaram $100 \%$ de suas sementes danificadas e $56 \%$ apresentaram pelo menos uma semente danificada.

TABELA 1: Características biométricas dos frutos de Hymenaea courbaril var. stilbocarpa e H. martiana. Valores médios e $( \pm)$ desvio padrão. Letras diferentes representam valores significativamente diferentes $(t=1,97)$.

\begin{tabular}{lcc}
\hline Variáveis & $\begin{array}{c}\text { H. courbaril var. } \\
\text { stilbocarpa }\end{array}$ & H. martiana \\
\hline Comprimento $(\mathrm{cm})$ & $11,6 \pm 2,5^{\mathrm{a}}$ & $10,2 \pm 2,3^{\mathrm{b}}$ \\
Largura $(\mathrm{cm})$ & $4,9 \pm 0,8^{\mathrm{a}}$ & $5,0 \pm 1,1^{\mathrm{a}}$ \\
Espessura (cm) & $3,9 \pm 0,6^{\mathrm{a}}$ & $3,7 \pm 0,7^{\mathrm{a}}$ \\
Sementes por fruto & $5,3 \pm 2,6$ & $3,3 \pm 1,9$ \\
Sementes Não Viáveis & $1,7 \pm 2,2$ & $2,1 \pm 1,7$ \\
\hline
\end{tabular}

Os frutos de $H$. martiana apresentaram, em média, $10,2 \mathrm{~cm}$ de comprimento, $5,0 \mathrm{~cm}$ de largura e $3,7 \mathrm{~cm}$ de espessura (Tabela 1). Foram encontradas de uma a nove $100 \%$ de suas sementes danificadas e $80 \%$ do total de frutos estudados apresentaram pelo menos uma semente danificada.

As sementes de $H$. courbaril var. stilbocarpa apresentaram em média $25,7 \mathrm{~mm}$ de comprimento; $18,7 \mathrm{~mm}$ de largura e $11,4 \mathrm{~mm}$ de espessura (Tabela 2 ). Para H. martiana os parâmetros biométricos descritos foram $32,4 \mathrm{~mm}, 23,3 \mathrm{~mm}$ e $16,3 \mathrm{~mm}$, respectivamente (Tabela 2).

TABELA 2: Características biométricas das sementes de Hymenaea courbaril var. stilbocarpa e $H$. martiana. Valores médios $(\mathrm{mm})$ e $( \pm)$ desvio padrão. Letras diferentes representam valores significativamente diferentes $(t=1,97)$.

\begin{tabular}{lcc}
\hline Variáveis & $\begin{array}{c}\text { H. courbaril var. } \\
\text { stilbocarpa }\end{array}$ & H. martiana \\
\hline Comprimento & $25,7 \pm 2,5^{\mathrm{b}}$ & $32,4 \pm 3,4^{\mathrm{a}}$ \\
Largura & $18,7 \pm 2,2^{\mathrm{b}}$ & $23,3 \pm 1,6^{\mathrm{a}}$ \\
Espessura & $11,4 \pm 2,1^{\mathrm{b}}$ & $16,3 \pm 2,3^{\mathrm{a}}$ \\
\hline
\end{tabular}

Os frutos maduros das duas espécies de Hymenaea apresentaram grande variação no peso, em $H$. courbaril var. stilbocarpa o valor médio encontrado foi $119,34 \mathrm{~g}$ (Tabela 3), já em H. martiana 62,85g (Tabela 3). As 
sementes apresentaram menor variação em relação à amplitude encontrada no peso dos frutos, em $\mathrm{H}$. courbaril var. stilbocarpa as sementes apresentaram peso médio de 5,71g (Tabela 3) e em H. martiana 7,29g (Tabela 3).

TABELA 3: Peso (g) médio e desvio padrão $( \pm)$ de frutos e sementes de $H$. courbaril var. stilbocarpa e H. martiana. Letras diferentes representam valores significativamente diferentes $(t=2,02)$.

\begin{tabular}{lcc}
\multicolumn{1}{c}{ Espécies } & Frutos & Sementes \\
\hline $\begin{array}{l}\text { H. courbaril var. } \\
\text { stilbocarpa }\end{array}$ & $119,34 \pm 34,32^{\mathrm{a}}$ & $5,71 \pm 1,77^{\mathrm{b}}$ \\
H. martiana & $62,85 \pm 50,88^{\mathrm{b}}$ & $7,29 \pm 1,88^{\mathrm{a}}$ \\
\hline
\end{tabular}

A análise comparativa entre as diferentes espécies do gênero Hymenaea mostrou que $H$. intermedia, encontrada na Floresta Amazônica, possui frutos grandes que se destacaram em todos os parâmetros biométricos comparados (Tabela 4). Com relação à biometria das sementes, H. martiana se destacou por apresentar os maiores valores para comprimento, largura e espessura (Tabela 5).

\section{Discussão}

A caracterização biométrica de frutos e sementes pode fornecer importantes subsídios para a diferenciação de espécies do mesmo gênero (CRUZ et al., 2001; DE-CARVALHO et al., 2005), principalmente aquelas que ocorrem na mesma região, como as espécies de Hymenaea estudadas. Morfologicamente, os frutos de H. courbaril var. stilbocarpa e H. martiana podem ser significativamente diferenciados em relação ao comprimento. Além disso, as sementes destas espécies foram estatisticamente distintas em relação ao comprimento, largura e espessura, parâmetros biométricos analisados.

Com base nos dados apresentados, os frutos de H. courbaril var. stilbocarpa foram caracterizados por serem maiores, possuírem maior número de sementes por fruto e significativamente mais pesados que os frutos de H. martiana. Por sua vez, as sementes de H. martiana são maiores, mais robustas e estatisticamente mais pesadas que as sementes de H. courbaril var. stilbocarpa.

As informações biométricas apresentadas são relevantes e ratificam a importância dos estudos morfológicos de frutos e sementes para a diferenciação de espécies. Estes dados serão úteis na identificação botânica, principalmente nos locais onde se recebe apenas frutos e sementes para análises de rotina (OLIVEIRA; PEREIRA, 1984).

As duas espécies de Hymenaea estudadas são árvores de médio a grande porte com grande potencial

TABELA 4: Parâmetros biométricos médios dos frutos de diferentes espécies do gênero Hymenaea.

\begin{tabular}{lcccl}
\multicolumn{1}{c}{ Espécies } & $\begin{array}{c}\text { Comprimento } \\
(\mathbf{c m})\end{array}$ & $\begin{array}{c}\text { Largura } \\
(\mathbf{c m})\end{array}$ & $\begin{array}{c}\text { Espessura } \\
(\mathbf{c m})\end{array}$ & \multicolumn{1}{c}{ Referências } \\
\hline H. stigonocorpa & 11,1 & 3,6 & 3,1 & Botelho et al., 2000 \\
H. stigonocarpa & 10,8 & 4,0 & - & De-Carvalho et al., 2005 \\
H. intermedia & 40,5 & 31,4 & 23,6 & Cruz et al., 2001 \\
H. courbaril var. stilbocarpa & 11,6 & 4,9 & 3,9 & Este estudo \\
H. martiana & 10,2 & 5,0 & 3,7 & Este estudo \\
\hline
\end{tabular}

TABELA 5: Parâmetros biométricos médios das sementes de diferentes espécies do gênero Hymenaea.

\begin{tabular}{lcccl}
\hline Espécies & $\begin{array}{c}\text { Comprimento } \\
(\mathbf{m m})\end{array}$ & $\begin{array}{c}\text { Largura } \\
(\mathbf{m m})\end{array}$ & $\begin{array}{c}\text { Espessura } \\
(\mathbf{m m})\end{array}$ & \multicolumn{1}{c}{ Referências } \\
\hline H. stigonocorpa & 18,9 & 16,4 & 14,4 & Botelho et al., 2000 \\
H. intermedia & 23,0 & 14,1 & 13,2 & Cruz et al., 2001 \\
H. courbaril var. stilbocarpa & 25,7 & 18,7 & 11,4 & Este estudo \\
H. martiana & 32,4 & 23,3 & 16,3 & Este estudo \\
\hline
\end{tabular}


paisagístico, pois produzem copas que oferecem boa sombra e frutos que atraem e alimentam a avifauna (MATOS; QUEIROZ, 2009: SILVA JÚNIOR; PEREIRA, 2009). Podem ser plantadas em avenidas, parques e praças (MATOS; QUEIROZ, 2009). Assim, os dados apresentados serão úteis para diferenciação destas espécies em campo facilitando a produção de mudas também para arborização urbana.

É importante ressaltar que $H$. martiana apresentou cerca de $50 \%$ dos frutos com $100 \%$ das sementes não viáveis. A viabilidade da semente foi avaliada com base nas características morfológicas do tegumento. Sugere-se a realização de estudos específicos relacionados aos fatores que afetam a viabilidade das sementes, pois estes podem comprometer o sucesso reprodutivo e consequente perpetuação da espécie no meio.

A análise comparativa das espécies de Hymenaea mostrou que os parâmetros biométricos devem ser levados em consideração na diferenciação de espécies do mesmo gênero. Hymenaea intermedia foi facilmente diferenciada das demais com base nos parâmetros biométricos relacionados ao fruto, já para H. martiana parâmetros biométricos relacionados às sementes foram mais eficientes para diferenciação.

Os dados apresentados destacaram variáveis biométricas de frutos e sementes, de fácil obtenção, que podem ser usadas na diferenciação de espécies do mesmo gênero que ocorrem em uma mesma região geográfica. Além disso, as informações apresentadas podem servir para nortear futuros estudos sobre a ecologia das espécies abordadas.

\section{Agradecimentos}

Ao Prof. Manoel Cláudio da Silva Júnior pela identificação do material botânico e sugestões ao longo do texto. Ao Gleidson Vítor pelo auxílio nas coletas de campo.

\section{Referências}

AQUINO, F. G.; WALTER, B. M. T.; RIBEIRO, J. F. Espécies Vegetais de Uso Múltiplo em Reservas Legais de Cerrado - Balsas,
MA. Revista Brasileira de Biociências, Porto Alegre, v. 5, supl. 1 p. 147-149, 2007.

BOTELHO, S. A.; FERREIRA, R. A.; MALAVASI, M. M.; DAVIDE, A. C. Aspectos morfológicos de frutos, sementes, plântulas e mudas de jatobá-do-cerrado (Hymenaea stigonocarpa Mart. ex Hayne) - Leguminosae Caesalpinioideae. Revista Brasileira de Sementes, Londrina, v. 22, p. 144-152, 2000.

COSTA-JUNIOR, C. L.; FIGUEIREDO, E. A emigração em Itapuranga nas décadas de 1980 e 1990. 2003. 35 f. Monografia (Graduação em Geografia) - Universidade Estadual de Goiás, Itapuranga. 2003.

CRUZ, E. D.; MARTINS, F. O.; CARVALHO, J. E. U. Biometria de frutos e sementes e germinação de jatobá-curuba (Hymenaea intermedia Ducke, Leguminosae-Caesalpinioideae). Revista Brasileira de Botânica, São Paulo, v. 24, n. 2, p. 161-165, 2001.

DE-CARVALHO, P. S.; MIRANDA, S. C.; SANTOS, M. L. Germinação e dados biométricos de Hymenaea stigonocarpa Mart. Ex Hayne (Leguminosae-Caesalpinoideae) - Jatobá-do-Cerrado. Revista Anhanguera, Goiânia, v. 6, n. 1, p. 101-121, 2005.

FEARNSIDE, P. M. Quantificação do serviço ambiental do carbono nas florestas amazônicas brasileiras. Oecologia Brasiliensis, Rio de Janeiro, v. 12, n. 4, p. 743-756, 2008.

FELFILI , J. M.; FAGG, C. W.; PINTO, J. R. R. Recuperação de áreas degradadas no Cerrado com espécies nativas do Bioma e de uso múltiplo para formação de corredores ecológicos e uso sustentável da reserva legal. In: FELFILI, J. M.; SAMPAIO, J. C.; CORREIA, C. R. M. A. Bases para a recuperação de áreas degradadas na Bacia do São Francisco. 1 ed. Brasília: CRAD/ Universidade de Brasília, 2008. p. 17-26.

FELFILI, J. M.; SILVA-JÚNIOR, M. C. Diversidade alfa e beta no cerrado sensu stricto, Distrito Federal, Goiás, Minas Gerais e Bahia. In: SCARIOT, A.; SOUSA-SILVA, J. C.; FELFILI, J. M. (Ed.). Cerrado: ecologia, biodiversidade e conservação. Brasília: Ministério do Meio Ambiente, 2005. p. 143-154.

FELFILI, J. M.; SILVA JÚNIOR, M. C.; SEVILHA, A. C.; FAGG, C. W.; WALTER, B. M. T.; NOGUEIRA, P. E.; REZENDE, A. V. Diversity, floristic and structural patterns of cerrado vegetation in Central Brazil. Plant Ecology, Dordrecht, v. 175, p. 37-46, 2004.

FORZZA, R. C.; BAUMGRATZ, J. F.; BICUDO, C. E. M.; CANHOS, D. A. L.; CARVALHO-JÚNIOR, A. A.; COSTA, A.; COSTA, D.; HOPKINS, M.; LEITMAN, P. M.; LOHMANN, L .G.; NIC-LUGHADHA, E.; MAIA, L. C.; MARTINELLI, G.; MENEZES, M.; MORIM, M. P.; COELHO, M. A. N.; PEIXOTO, A. L.; PIRANI, J. R.; PRADO, J.; QUEIROZ, L. P.; SOUZA, S.; SOUZA, V. C.; STEHMANN, J. R.; SYLVESTRE, L. S.; WALTER, B. M. T.; ZAPPI, D. Síntese da diversidade brasileira. In: FORZZA, R. C.; LEITMAN, P. M.; COSTA, A.; CARVALHO JÚNIOR, A. A.; PEIXOTO, A. L.; WALTER, B. M. T.; BICUDO, C.; ZAPPI, D.; COSTA, D. P.; LLERAS, E.; MARTINELLI, G.; LIMA, H. C.; PRADO, J.; STEHMANN, J. R.; BAUMGRATZ, J. F. A.; PIRANI, J. R.; SYLVESTRE, L. S.; MAIA, L. C.; LOHMANN, L. G.; PAGANUCCI, L.; SILVEIRA, M.; NADRUZ, M.; MAMEDE, M. C. H.; BASTOS, M. N. C.; MORIM, M. P.; BARBOSA, M. R.; MENEZES, M.; HOPKINS, M.; SECCO, R.; CAVALCANTI, T.; SOUZA, V. C. Catálogo de Plantas e Fungos do Brasil. v. 1. Rio de Janeiro: Instituto de Pesquisas Jardim Botânico do Rio de Janeiro, 2010. p. 21-39. 
FURLEY,P.A.; RATTER, J.A. Soil resources and plant communities of the central Brazilian cerrado and their development. Journal of Biogeography, New York, v. 15, n. 1, p. 97-108, 1988.

KLINK, C. A.; MACHADO, R. B. Conservation of the Brazilian Cerrado. Conservation Biology, San Francisco, v. 19, p. 3, p. 707 $713,2005$.

LEWINSOHN, T. M.; PRADO, P. I. Biodiversidade brasileira: síntese do estado atual do conhecimento. São Paulo: Contexto, 2002. $176 \mathrm{p}$.

LORENZI, H. Árvores brasileiras: manual de identificação e cultivo de plantas arbóreas do Brasil. 5 ed. Nova Odessa: Instituto Plantarum, 2008. 384 p.

MATOS, E.; QUEIROZ, L. P. Árvores para cidades. Salvador: Ministério Público do Estado da Bahia/Solisluna, 2009. 344 p.

MENDONÇA, R. C.; FELFILI, J. M.; WALTER, B. M. T.; SILVA JÚNIOR, M. C.; REZENDE, A. V.; FILGUEIRA, T. S.; NOGUEIRA, P. E. Flora vascular do Cerrado. In: SANO, S. M.; ALMEIDA, S. P.; RIBEIRO, J. F. Cerrado: ecologia e flora. Brasília: Embrapa Cerrados, 2008. p. 15-90.

MITTERMEIER, R. A.; ROBLES, P.; HOFFMAN, M.; PILGRIM, J.; BROOKS, T.; MITTERMEIER, C. G.; LAMOREUX, J.; FONSECA, G. B. Hotspots revisited: earth's biologically richest and most endangered ecoregions. Mexico City: Conservation International, 2005. $96 \mathrm{p}$.

MMA/IBAMA/PNUD. Relatório técnico de monitoramento do desmatamento no bioma Cerrado, 2002 a 2008. Dados Revisados. Acordo de Cooperação técnica. Brasília: Centro de Sensoriamento Remoto - CSR/IBAMA, 2009. 69 p.

OLIVEIRA, E. C.; PEREIRA, T. S. Morfologia dos frutos alados em Leguminosae-Caesalpinioideae - Martiodendron Gleason, Peltophorum (Vogel) Walpers, Sclerolobium Vogel, Tachigalia Aublet e Schizolobium Vogel. Rodriguesia, Rio de Janeiro, v. 36, n. 60, p. $35-42,1984$.
OLIVEIRA-FILHO, A. T.; RATTER, J. A. Vegetation physiognomies and woody flora of the Cerrado biome. In: OLIVEIRA, O. S.; MARQUIS, R. J. (Eds.). The cerrados of Brazil: ecology and natural history of a neotropical savanna. New York: Columbia University Press, 2002. p. 91-120.

RIBEIRO, J. F.; BRIDGEWATER, S.; RATTER, J. A.; SOUSASILVA, J. C. Ocupação do bioma Cerrado e conservação da sua diversidade vegetal. In: SCARIOT, A.; SOUSA-SILVA, J. C.; FELFILI, J. M. Cerrado: Ecologia, Biodiversidade e Conservação. Brasília: Ministério do Meio Ambiente, 2005. p. 385-399.

RIBEIRO. J. F.; WALTER. B. M. T. As principais fitofisionomias do bioma Cerrado. In: SANO, S. M.; ALMEIDA, S. P.; RIBEIRO, J. F. Cerrado: ecologia e flora. Brasília: Embrapa Cerrados, 2008. p. $153-212$.

RIBEIRO, R. D. Hymenaea. In: Lista de Espécies da Flora do Brasil. Jardim Botânico do Rio de Janeiro, 2010. Disponível em $<$ http://floradobrasil.jbrj.gov.br/2010/FB083198>.

SANO, E. E.; ROSA, R.; BRITO, J. L. S.; FERREIRA, L. G. Mapeamento semidetalhado do uso da terra do Bioma Cerrado. Pesquisa Agropecuária Brasileira, Brasília, v. 43, n. 1, p. 153156, 2008.

SANO, E. E.; ROSA, R.; BRITO, J. L. S.; FERREIRA, L. G. Land cover mapping of the tropical savana region in Brazil. Environmental Monitoring and Assessment, Dordrecht, v. 166, p. 113-124, 2010.

SILVA JÚNIOR, M. C.; PEREIRA, B. A. S. + Cem árvores do Cerrado: Matas de Galeria. Guia de Campo. Brasília: Rede de Sementes do Cerrado, 2009. 278 p. 